\title{
A case of sudden death after chemotherapy in a cancer of unknown primary patient with idiopathic pulmonary arterial hypertension
}

\author{
Masayuki Takeda $\cdot$ Yoshikazu Hasegawa $\cdot$ Keisuke Shioji • Masahiro Kawashima • \\ Koki Miura • Satoshi Marumo • Yoshihiko Koshimo • Takehiko Kobayashi • \\ Takashi Teranishi $\cdot$ Yuichi Higami $\cdot$ Motokazu Kato
}

Received: 14 February 2012/ Accepted: 15 April 2012

(C) The Japan Society of Clinical Oncology 2012

\begin{abstract}
We here describe the first example of a cancer of unknown primary (CUP) patient with concomitant idiopathic pulmonary arterial hypertension who was treated with docetaxel monotherapy. A 43-year-old woman was admitted to our hospital with left chest pain in June 2011. Based on the results of the general physical and other examinations, the patient was diagnosed as having CUP. The patient was treated with total doses of $40 \mathrm{~Gy}$ to a painful bone metastasis, followed by docetaxel monotherapy at $60 \mathrm{mg} / \mathrm{m}^{2}$. Nine days after chemotherapy, she developed shortness of breath, and laboratory investigations showed a substantial increase in serum amino-terminal pro-brain natriuretic peptide levels of $293.2 \mathrm{pg} / \mathrm{ml}$ (normal range, $<125 \mathrm{pg} / \mathrm{ml}$ ). At day 17 , the right ventricular systolic pressure increased from 93 to $125 \mathrm{mmHg}$, suggesting right-sided heart failure, and consequently she died on day 21.
\end{abstract}

Keywords Docetaxel - Pulmonary arterial hypertension . Cancer of unknown primary

\footnotetext{
M. Takeda $(\bowtie) \cdot$ Y. Hasegawa

Department of Medical Oncology, Kishiwada City Hospital,

1001 Gakuhara-cho, Kishiwada, Osaka 596-8501, Japan

e-mail: takedamasa2004@yahoo.co.jp

K. Shioji

Department of Cardiology, Kishiwada City Hospital,

1001 Gakuhara-cho, Kishiwada, Osaka 596-8501, Japan

M. Kawashima - K. Miura - S. Marumo - Y. Koshimo ·

T. Kobayashi · T. Teranishi · Y. Higami · M. Kato

Department of Respirology, Kishiwada City Hospital,

1001 Gakuhara-cho, Kishiwada, Osaka 596-8501, Japan
}

\section{Introduction}

Despite the development of excellent diagnostic tools, there are still patients with metastatic cancer in whom the site of the primary tumor cannot be determined; these patients are defined as having cancer of unknown primary (CUP). Among the new agents that became available in the 1990s, the taxanes were reported to show significant activity against CUP. It is still unclear whether chemotherapy can be safely administered to CUP patients with concomitant idiopathic pulmonary arterial hypertension (IPAH). We here describe the first example of a CUP patient with concomitant IPAH who was treated with docetaxel monotherapy.

\section{Case presentation}

The patient was a 43-year-old woman who presented with unexplained dyspnea at age 15 in 1983. A thorough evaluation revealed idiopathic pulmonary arterial hypertension (IPAH) as the etiology. In 2005, she was started on continuous intravenous epoprostenol therapy and continued for 6 years without developing right-sided heart failure. She also received oxygen therapy and routine prophylactic anticoagulation with warfarin, and her baseline oxygen saturation was above $95 \%$ on $4 \mathrm{l} / \mathrm{m} \mathrm{O}_{2}$ (nasal); the target international normalized ratio (INR) had been stable and within the therapeutic range of 2.0-3.0. She was admitted to our hospital with left chest pain in June 2011. Chest computed tomography showed bilateral pulmonary nodules (Fig. 1a), and positron emission tomography/computed tomography (PET/CT) imaging revealed intense 18 F-fluorodeoxyglucose uptake in the lesions corresponding to the bilateral lungs and left sixth rib (Fig. 1b). Ultrasound 
Fig. 1 Chest computed tomography showed bilateral pulmonary nodules (a), and positron emission tomography/ computed tomography (PET/ CT) imaging revealed intense 18 F-fluorodeoxyglucose uptake in the lesions corresponding to the bilateral lungs and left sixth rib (b)
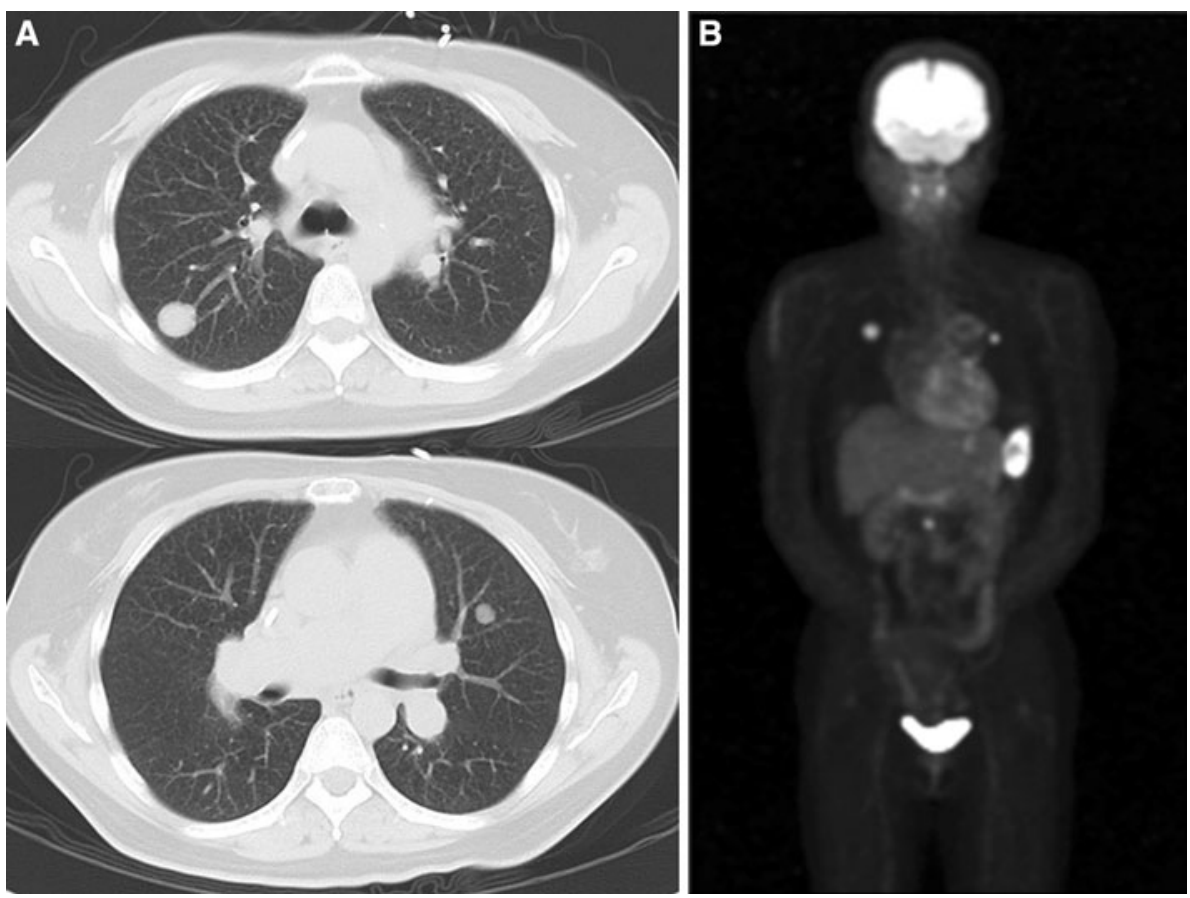

scan and endoscopic examination did not allow identification of the site of the primary lesion. A biopsy specimen from a rib showed a poorly differentiated carcinoma, but the immunohistochemical examination failed to define a primary tumor site. Based on the results of the general physical and other examinations, the patient was diagnosed as having cancer of unknown primary (CUP). The patient was treated with total doses of 40 Gy at 2.5 Gy per fraction to a painful bone metastasis, followed by docetaxel monotherapy at $60 \mathrm{mg} / \mathrm{m}^{2}$. Radiation therapy provided significant relief from the painful bone metastases, and no significant radiation therapy-related complications were found. Nine days after the administration of the first cycle of docetaxel, she developed shortness of breath without a cough, fever or chest pain, and a chest X-ray did not show any infiltrate, consolidation or effusion, but did reveal an enlarged heart and bilateral pulmonary nodules consistent with metastases. Laboratory investigations showed grade 1 anemia (hemoglobin $10.1 \mathrm{~g} / \mathrm{dl}$ ), grade 4 neutropenia (220/ $\mu \mathrm{l})$, grade 1 thrombocytopenia $(77,000 / \mu \mathrm{l})$ and a substantial increase in serum amino-terminal pro-brain natriuretic peptide (NT-proBNP) levels of $293.2 \mathrm{pg} / \mathrm{ml}$ (normal range, $<125 \mathrm{pg} / \mathrm{ml}$ ); however, there were no episodes of febrile neutropenia or bleeding. The patient received prophylactic filgrastim once daily for 4 days. At day 17, her shortness of breath gradually worsened without a cough, fever or chest pain; however, a chest X-ray did not show any infiltrate, consolidation or effusion. Oxygen saturation dropped below $90 \%$ on $4 \mathrm{l} / \mathrm{m} \mathrm{O}_{2}$ (nasal), and nasal cannula oxygen was replaced by an oxygen mask. Laboratory investigations showed grade 2 anemia (hemoglobin $9.5 \mathrm{~g} / \mathrm{dl}$ ),
Table 1 Changes in clinical parameters

\begin{tabular}{lllr}
\hline & \multicolumn{3}{l}{ Time course under docetaxel } \\
\cline { 2 - 4 } & Pre-treatment & Day 9 & Day 17 \\
\hline RVSP (mmHg) & 93 & - & 125 \\
NT-proBNP (pg/ml) & 86.3 & 293.2 & 2249 \\
WHO/NYHA FC & II & III & IV \\
\hline
\end{tabular}

RVSP right ventricular systolic pressure, $N T$-proBNP amino-terminal pro-brain natriuretic peptide, WHO/NYHA FC World Health Organization/New York Heart Association functional class

normalization in neutrophils $(10,137 / \mu \mathrm{l})$, grade 3 thrombocytopenia $(36,000 / \mu \mathrm{l})$ and dramatically increased NTproBNP levels of $2,249 \mathrm{pg} / \mathrm{ml}$. The fibrin degradation product (FDP) was at the normal level of $4.9 \mu \mathrm{g} / \mathrm{ml}$ (normal range, $0.1-4.99 \mu \mathrm{g} / \mathrm{ml}$ ), and the INR was 2.15 , a value well within the targeted range. The electrocardiogram showed right ventricular hypertrophy, but no arrhythmias. Right ventricular systolic pressure (RVSP) measured by echocardiography increased from 93 to $125 \mathrm{mmHg}$ (Table 1), suggesting right-sided heart failure, but left ventricular (LV) systolic function was normal with an ejection fraction (EF) of $86 \%$. Furosemide was additionally supplied, but her condition worsened and she died of respiratory failure on day 21 .

\section{Discussion}

Neither standard nor optimal chemotherapeutic regimens have yet been determined because of the complex and 
heterogeneous presentations of patients with CUP. Among the new agents that became available in the 1990s, the taxanes were reported to show significant activity against CUP. Based on the results of several studies, it appears that platinum compounds plus taxanes may prove to be as effective or more effective than previous regimens [1, 2]. It is still unclear whether chemotherapy can be safely administered to CUP patients with concomitant IPAH. Our present patient was treated with docetaxel monotherapy to avoid the risk of fluid overload from platinum-based chemotherapy, resulting in right ventricular failure; however, she suffered chemotherapy-related early death due to PAH exacerbation. Several factors may be responsible for this, including thromboembolism, tumor microemboli and damage to blood vessels in the lungs. Although patients with cancer have a 4- to 6-fold increased risk of thrombosis compared with the general population [3], the risk of thrombosis in the present case seemed to be low because the target international normalized ratio (INR) in this IPAH patient treated with warfarin had been stable and within the therapeutic range of 2.0-3.0, and the FDP values were normal during a course of docetaxel therapy. However, the possibility of thrombosis should be considered because contrast-enhanced CT was not performed. Pulmonary hypertension caused by tumor cell microembolism is a rare occurrence and an extremely difficult diagnosis to make prior to death [4]. Tumor emboli needed to be included in the differential diagnosis in the present case; however, it seems unlikely that tumor microembolism occurred early in the course of chemotherapy without evidence of rapid tumor progression. The other possible explanation is that docetaxel induced pulmonary hypertension through modification of endothelial function. Docetaxel has been proven to have antiangiogenic activity, and its resulting impaired angiogenesis or endothelial dysfunction may be the mechanism of elevated pulmonary blood pressure [5], but whether its antiangiogenic properties are linked to pulmonary arterial hypertension or not remains unclear. Moreover, an autopsy was not performed in this case, and the information is insufficient to draw a definitive conclusion on the effects of docetaxel on idiopathic pulmonary arterial hypertension.
In conclusion, taxanes may be one of the treatment options for patients with CUP when alternative therapeutic options are limited. Since chemotherapy is considered to be one of the risk factors for developing secondary PAH $[6,7]$ and exacerbation of pulmonary arterial hypertension, which might have been related to chemotherapy, was found in the present case, it should be emphasized that chemotherapy may increase treatment-related mortality in IPAH patients harboring cancer. Further investigations are also required to understand whether pulmonary hypertension can be considered as an adverse effect of docetaxel and how the drug eventually affects vessel endothelial cells.

Conflict of interest All authors declare no actual or potential conflicts of interest.

\section{References}

1. Greco FA, Erland JB, Morrissey LH et al (2000) Carcinoma of unknown primary site: phase II trials with docetaxel plus cisplatin or carboplatin. Ann Oncol 11:211-215

2. Golfinopoulos V, Pentheroudakis G, Salanti G et al (2009) Comparative survival with diverse chemotherapy regimens for cancer of unknown primary site: multiple-treatments meta-analysis. Cancer Treat Rev 35:570-573

3. Blom JW, Doggen CJ, Osanto S, Rosendaal FR et al (2005) Malignancies, prothrombotic mutations, and the risk of venous thrombosis. JAMA 293:715-722

4. Soares FA, Pinto AP, Landell GA et al (1993) Pulmonary tumor embolism to arterial vessels and carcinomatous lymphangitis. A comparative clinicopathological study. Arch Pathol Lab Med 117: 827-831

5. Sweeney CJ, Miller KD, Sissons SE et al (2001) The antiangiogenic property of docetaxel is synergistic with a recombinant humanized monoclonal antibody against vascular endothelial growth factor or 2-methoxyestradiol but antagonized by endothelial growth factors. Cancer Res 61:3369-3372

6. Bentur L, Cullinane C, Wilson P et al (1991) Fatal pulmonary arterial occlusive vascular disease following chemotherapy in a 9-month-old infant. Hum Pathol 22:1295-1298

7. Limsuwan A, Pakakasama S, Rochanawutanon M et al (2006) Pulmonary arterial hypertension after childhood cancer therapy and bone marrow transplantation. Cardiology 105:188-194 